\title{
Tromboprofilaxia na artroplastia total do joelho*
}

\section{Thromboprophylaxis for Total Knee Arthroplasty}

\author{
Julio Cesar Gali ${ }^{1 \odot ~ D a n i l o ~ B o r d i n i ~ C a m a r g o ~}{ }^{1}$ \\ ${ }^{1}$ Departamento de Ortopedia, Faculdade de Ciências Médicas e da \\ Saúde, Pontifícia Universidade Católica de São Paulo (PUC-SP), \\ Sorocaba, SP, Brasil \\ Rev Bras Ortop 2019;54:1-5.
}

\begin{abstract}
Address for correspondence Julio Cesar Gali, MD, PhD, Departamento de Ortopedia, Faculdade de Ciências Médicas e da Saúde, Pontifícia Universidade Católica de São Paulo (PUC-SP), Sorocaba, SP 18030-070, Brasil (e-mail: jcgali@pucsp.br).
\end{abstract}

\section{Resumo \\ Palavras-chave \\ - prótese total de joelho \\ - complicações \\ - trombose venosa \\ - prevenção}

Os autores descrevem uma atualização dos principais métodos de prevenção da trombose venosa profunda após artroplastia total do joelho, classificados em métodos mecânicos e farmacológicos. Reportam as principais drogas usadas, dosagem, riscos e benefícios comparativos.
Abstract
Keywords
- total knee replacement
- complications
- venous thrombosis
- prevention

The authors report an update of the main methods for preventing deep vein thrombosis after total knee replacement, which are divided into mechanical and pharmacological methods. The current principal used drugs, their dosages, and the comparative risks and benefits are also reported.

\section{Introdução}

A artroplastia total de joelho (ATJ) é um procedimento cirúrgico seguro para alívio da dor e melhoria de limitações funcionais causadas pela artrose severa, nos casos em que o tratamento clínico não é mais efetivo. Entretanto, algumas complicações podem ocorrer. Uma complicação potencial é a trombose venosa profunda (TVP). Existem alguns fatores de risco para a ocorrência da TVP: idade acima de 60 anos, obesidade, uso de contraceptivos orais ou por adesivos,

\footnotetext{
* Trabalho desenvolvido no Departamento de Ortopedia, Faculdade de Ciências Médicas e da Saúde, Pontifícia Universidade Católica de São Paulo (PUC-SP), Sorocaba, SP, Brasil. Publicado originalmente por Elsevier Editora Ltda. (c) 2018 Sociedade Brasileira de Ortopedia e Traumatologia.

(D) Julio Cesar Gali's ORCID is https://orcid.org/0000-0003-07521266.
}

received

June 4, 2017

accepted

June 27, 2017

published online

December 1, 2017 terapia de reposição hormonal, veias varicosas, doença inflamatória intestinal, histórico de TVP ou embolia pulmonar (EP), histórico familiar de trombose e tempo prolongado de garroteamento, se esse for usado na artroplastia.

Song et al. fizeram um estudo observacional prospectivo com flebografia bilateral de membros inferiores em 109 pacientes, dentro de uma semana após a ATJ unilateral primária. Eles relataram que a incidência de TVP sintomática e TVP assintomática, após o procedimento cirúrgico, foi de 4,6 e $18,3 \%$, respectivamente. ${ }^{1}$

Basicamente, existem os métodos mecânicos e farmacológicos usados para prevenção de TVP.

\section{Métodos Mecânicos}

A mobilização precoce do paciente é a medida mais simples e barata para prevenir a formação de trombos. Existem outras
DOI https://doi.org/

10.1016/j.rbo.2017.06.025. ISSN 0102-3616.
Copyright $\odot 2019$ by Sociedade Brasileira License terms de Ortopedia e Traumatologia. Published by Thieme Revnter Publicações Ltda, Rio de Janeiro, Brazil 
modalidades de prevenção da TVP por métodos mecânicos. A compressão pneumática intermitente é usada para diminuir a estase venosa, aumentar a velocidade do fluxo sanguíneo e aumentar o nível de fibrinolisinas circulantes. As bombas venosas podais podem simular a ação fisiológica de bombeamento sobre o plexo venoso que ocorre ao ficar de pé e durante a caminhada e, portanto, podem aumentar o fluxo venoso. Meias de compressão graduada promovem suave pressão nas pernas para prevenir o acúmulo de sangue.

Contudo, a compressão mecânica é geralmente menos eficaz para diminuir a prevalência da TVP do que os métodos farmacológicos. Os métodos mecânicos podem ser usados em pacientes com risco elevado de hemorragia ou combinados com métodos farmacológicos.

Blanchard et al. avaliaram a ocorrência de TVP em 108 pacientes após ATJ, com flebografia feita oito a 12 dias após a cirurgia. A prevenção da TVP foi feita em 60 deles com heparina de baixo peso molecular (HBPM) e em 48 a prevenção mecânica foi feita com a compressão pneumática intermitente do pé. Quarenta e sete TVPs foram diagnosticadas em todos os pacientes, 16 (26,7\%) no grupo de HBPM e 31 (64,6\%) no grupo de profilaxia mecânica. A diferença entre os dois grupos foi considerada altamente significativa $(p<0,001){ }^{2}$

Lachiewicz et al., em um estudo prospectivo randomizado, compararam dois métodos de compressão da panturrilha para profilaxia de tromboembolismo após a ATJ: uma compressão assimétrica com insuflação rápida (CAIR) e um dispositivo circunferencial sequencial (DCS). Após artroplastia total unilateral primária, a incidência de trombos foi de $8,4 \%$ para o grupo CAIR e $16,8 \%$ para o grupo DCS $(p=0,03)$. A incidência de trombos em pacientes com ATJ bilateral foi de $4 \%$ para o grupo CAIR, em comparação com $22,7 \%$ para o grupo DCS ( $p=0,05$ por joelho). Eles concluíram que a CAIR apresentou uma diminuição significativa da taxa de tromboembolismo. ${ }^{3}$

He et al., em um estudo de metanálise, demonstraram a ausência de eficácia da terapia de movimento passivo contínuo na prevenção de doença tromboembólica venosa profunda, em pacientes após ATJ. ${ }^{4}$

\section{Métodos Farmacológicos}

Algumas especialidades médicas tentaram criar um guia clínico prático para prevenir a ocorrência de TVP. O primeiro foi feito pelo American College of Chest Physicians (ACCP), em 1985. Esse guia tinha dois níveis de recomendação. O mais eficaz foi baseado em ensaios clínicos randomizados com resultados consistentes. Os fármacos que corresponderam a essas indicações foram a varfarina, com uma relação internacional normalizada (INR) de 2 a 3 , a heparina de baixo peso molecular e o fondaparinux.

Por outro lado, existe a preocupação de que INR de 2 a 3 pode ser elevado para cirurgias ortopédicas e o uso de drogas indicadas para obter esse nível, independentemente do perfil de risco dos pacientes, poderia colocar alguém com baixo risco relativo de TVP em um risco elevado de sangramento. ${ }^{5}$ Havia também uma correlação muito baixa entre a presença de TVP e a ocorrências de EP e, ainda, o significado da TVP assintomática foi questionado. ${ }^{6}$

Em 2012, a American Academy of Orthopaedic Surgeons (AAOS) publicou uma diretriz sobre a prevenção de TVP em pacientes submetidos à artroplastia eletiva de quadril e joelho. Foi relatado que esses pacientes estão em risco de hemorragia e complicações associadas ao sangramento. Sua recomendação foi o uso de agentes farmacológicos e/ou dispositivos mecânicos de compressão para a prevenção de TVP em pacientes que não têm risco elevado para tromboembolismo ou sangramento venoso, além do procedimento cirúrgico propriamente dito. Para pacientes com histórico de TVP prévio estão indicados a profilaxia farmacológica e dispositivos de compressão mecânica; já para aqueles com distúrbio hemorrágico conhecido e/ou doença hepática ativa, sugere-se o uso de dispositivos de compressão mecânica, apenas. ${ }^{7}$

As drogas prescritas para prevenir a formação ou o crescimento de trombos são chamadas antitrombóticas e consistem em fármacos antiplaquetários e anticoagulantes.

A aspirina é uma droga antiplaquetária eficaz. Em 2006, Lotke e Lonner publicaram seus resultados com o uso da aspirina combinada com mobilização precoce, anestesia regional e bombas podais para a prevenção de eventos tromboembólicos em 3.473 pacientes submetidos a ATJ. A prevalência de EP não fatal e trombose venosa proximal foi de $0,26 \%$ e $0,2 \%$, respectivamente. Eles concluíram que a aspirina é mais segura e igualmente eficaz do que outros agentes quimioprofiláticos para a prevenção de TVP após ATJ. ${ }^{8}$

Callaghan et al., em 2008, relataram que a incidência de TVP em uma população de ATJ de baixo risco foi de $2,6 \%$ com o uso profilático de aspirina, deambulação precoce e bombas podais. Eles consideraram a eficácia da prevenção extremamente bem-sucedida. ${ }^{9}$

Em 2010, Bozic et al. compararam a aspirina com a varfarina ou HBPM para a prevenção do tromboembolismo venoso em pacientes com ATJ. A ocorrência de TVP ou EP entre pessoas tratadas com aspirina foi de $2,3 \%$, comparada a $3,1 \%$ para os pacientes do grupo de HBPM e $4 \%$ para os indivíduos do grupo da varfarina ( $p=0,0037$ para a aspirina versus HBPM e $p<0,001$ para a aspirina versus varfarina). ${ }^{10}$

A prescrição de aspirina recomendada é de $325 \mathrm{mg}$ duas vezes ao dia. No entanto, uma publicação recente relatou que $81 \mathrm{mg}$ duas vezes ao dia não é inferior à dosagem anteriormente recomendada de aspirina para profilaxia de tromboembolismo venoso, após artroplastia total. ${ }^{11}$

Os cumarínicos (varfarina) são antagonistas da vitamina $\mathrm{K}$ (droga anticoagulante oral). Existem algumas desvantagens do uso da varfarina: muito tempo para início da ação, meiavida longa, necessidade de controle do INR e a interação entre cumarínicos e dieta.

As HBPM são fármacos anticoagulantes que têm elevada atividade antifator Xa e baixa atividade anti-Ila ou antitrombina. Liu et al. avaliaram dois protocolos para prevenção de TVP com 40 mg de enoxaparina subcutânea, depois de ATJ. Um grupo de pacientes iniciou o uso da medicação 12 horas após o fechamento da ferida, o outro, 24 horas após. 0 tratamento foi mantido por 10 a 14 dias. Os dois regimes 
tiveram resultados similares para prevenção de TVP, mas o grupo que iniciou o tratamento 24 horas após o fechamento da incisão cirúrgica apresentou resultados mais seguros quanto ao sangramento $(p<0,05) .^{12}$

Arsoy et al. compararam o uso de HBPM com compressão mecânica e aspirina, depois de próteses totais de quadril ou joelho. Concluíram que o uso dos últimos diminuiu as taxas de reinternação, grandes complicações e problemas com o ferimento, depois de artroplastias totais primárias. ${ }^{13}$

O fondaparinux é um pentassacarídeo sintético inibidor específico do fator Xa. Bauer et al., em um estudo duplo cego, compararam doses subcutâneas de $2,5 \mathrm{mg}$ de fondaparinux com $30 \mathrm{mg}$ de enoxaparina duas vezes por dia, em pacientes submetidos a grandes cirurgias eletivas de joelho. No 11。 dia o grupo tratado com fondaparinux apresentou uma incidência significativamente menor de tromboembolismo venoso $(12,5 \%)$ comparado com o grupo tratado com enoxaparina (27,8\%), correspondeu a uma redução de 55,2\% no risco $(p<0,001)$, mas um sangramento maior e significativo ocorreu no grupo do fondaparinux $(p=0,006) .{ }^{14}$

A rivaroxabana é um inibidor direto do fator Xa. Num estudo randomizado duplo-cego, Lassen et al. compararam a rivaroxabana oral, na dosagem de $10 \mathrm{mg}$ uma vez ao dia, administrada seis a oito horas após a cirurgia, com enoxaparina subcutânea, na dosagem de $40 \mathrm{mg}$ uma vez por dia, prescrita 12 horas antes da cirurgia, em 2.531 pacientes submetidos à ATJ. Um tromboembolismo venoso maior ocorreu em 1,0\% dos pacientes do grupo da rivaroxabana e em 2,6\% dos pacientes do grupo da enoxaparina (redução do risco absoluto, $1,6 \% ; p=0,01$ ). Ocorreu sangramento importante em $0,6 \%$ dos pacientes tratados com rivaroxabana e em $0,5 \%$ dos tratados com enoxaparina. ${ }^{15}$

A rivaroxabana foi comparada à enoxaparina nos estudos Record. Ocorreu menos sangramento no local cirúrgico nas ATJ com o uso da rivaroxabana, mas esse foi semelhante nas próteses totais de quadril. ${ }^{16}$

A dabigatrana é um inibidor direto da trombina. A dosagem preconizada é de $110 \mathrm{mg}$, uma a quatro horas após a cirurgia; depois $110 \mathrm{mg}$, duas vezes por dia, durante 10 dias, para a ATJ. Em um estudo com 1.728 pacientes submetidos à substituição articular primária, o uso da dabigatrana levou a um aumento de $20 \%$ no sangramento da ferida em comparação com um aumento de 5\% encontrado em um regime multimodal, composto de HBPM durante a internação e aspirina por tempo estendido ( $p<0,001)$. A taxa de tromboembolismo no grupo de pacientes com dabigatrana foi de $1,3 \%$ em comparação com $0,3 \%$ nos pacientes que receberam o esquema de tromboprofilaxia multimodal $(p=0,047) \cdot{ }^{17}$

Os resultados dos ensaios clínicos RE-Novate, RE-Model e RE-Mobilize avaliaram o uso da dabigatrana, tanto no regime europeu (40 mg/dia) e americano (30 mg a cada 12 horas), nas cirurgias de grande porte do quadril e joelho e mostraram resultados não inferiores àqueles obtidos com a enoxaparina para prevenção da TVP. ${ }^{18}$

A apixabana é um inibidor do fator Xa. A dose sugerida é de 2,5 mg duas vezes por dia, começa 12 a 24 horas após a operação, continuada durante 12 dias ( \pm 2 ) após ATJ e 35 dias ( \pm 3 ) após artroplastia total de quadril. Raskob et al.
Fizeram uma análise combinada de dois estudos randomizados duplo-cegos previamente reportados que incluiu 8.464 pacientes, compararam $2,5 \mathrm{mg}$ de apixabana, duas vezes por dia, e $40 \mathrm{mg}$ de enoxaparina diária. Um tromboembolismo venoso maior ocorreu em $0,7 \%$ e $1,5 \%$ nos pacientes tratados com apixabana e enoxaparina, respectivamente (diferença de risco, apixabana menos enoxaparina $=-0,8 \%, p=0,001$ para superioridade). Sangramentos maiores aconteceram em $0,7 \%$ e $0,8 \%$ nos pacientes tratados com apixabana e enoxaparina, respectivamente (diferença de risco $-0,02 \%)$. Sangramentos maiores somados aos clinicamente relevantes não maiores ocorreram em $14,4 \%$ dos pacientes que receberam a apixabana e em $4,9 \%$ dos pacientes tratados com enoxaparina (diferenc, a de risco - 0,6\%). Eles concluíram que a apixabana é mais eficaz do que a enoxaparina, sem aumentar o sangramento. ${ }^{19}$

Uma revisão sistemática de metanálise e comparação indireta de tratamento confrontou o uso da rivaroxabana, apixabana e dabigatrana versus a enoxaparina para profilaxia contra TVP após artroplastia total de quadril ou joelho. Os riscos relativos e seus respectivos intervalos de confiança de 95\% foram calculados para cada estudo e para os estudos combinados, para cada um dos anticoagulantes. Os autores relataram que o risco relativo de sangramento clinicamente relevante foi maior com rivaroxabana, semelhante com o dabigatrano e menor com apixabana. Comparado com a enoxaparina, o risco de tromboembolismo venoso sintomático foi menor com a rivaroxabana e similar com a dabigatrana e a apixabana. ${ }^{20}$

Por outro lado, Revankar et al., em uma avaliação econômica do uso de apixabana, mostrou que essa droga é uma opção benéfica para a prevenção de TVP pós-cirúrgico, em comparação com a enoxaparina. ${ }^{21}$

A edoxabana é um inibidor direto oral do fator Xa. $\mathrm{O}$ estudo STAR E-3 comparou $30 \mathrm{mg}$ de edoxabana uma vez ao dia, com início seis a 24 horas após a cirurgia, ou enoxaparina 20 mg, subcutâneo, duas vezes ao dia, começa 24 a 36 horas após a cirurgia por 11 a 14 dias depois de ATJ, em pacientes no Japão e Taiwan. Embolia pulmonar e TVP sintomáticas ou TVP assintomáticas ocorreram em 7,4\% dos que receberam edoxabana e $13,9 \%$ dos pacientes que receberam enoxaparina (redução do risco relativo, 46,8\%), demonstrou não inferioridade $(p<0,001)$ e superioridade $(p=0,01)$ da edoxabana relativo à enoxaparina. A incidência de todos os eventos hemorrágicos (sangramentos maiores, sangramentos maiores clinicamente não relevantes e sangramentos menores) foi de $22,3 \%$ e $18,9 \%$, nos grupos de tratamento com edoxabana e enoxaparina, respectivamente $(p=0,265)$, sugeriu que a eficácia superior da edoxabana não foi associada a uma maior incidência de eventos hemorrágicos. ${ }^{22} \mathrm{~A}$ betrixabana é um inibidor direto oral do fator Xa. Seu uso, nas dosagens de $15 \mathrm{mg}$ duas vezes ao dia e $40 \mathrm{mg}$ duas vezes ao dia, foi comparado a $30 \mathrm{mg}$ de enoxaparina duas vezes ao dia, no ensaio clínico Expert. A incidência de TVP para a betrixabana $15 \mathrm{mg}$ duas vezes ao dia, betrixabana $40 \mathrm{mg}$ duas vezes ao dia e enoxaparina duas vezes ao dia foi de $20 \%, 15 \%$ e $10 \%$, respectivamente. Não houve relato de sangramentos com 0 betrixabana $15 \mathrm{mg}$ duas vezes ao dia. Com o uso da 
betrixabana, na dosagem de $40 \mathrm{mg}$ duas vezes ao dia, ocorreu sangramento em $2,4 \%$ dos casos. Já com uso da enoxaparina aconteceram $4,5 \%$ de sangramentos não maiores clinicamente significantes e $2,3 \%$ de sangramentos maiores clinicamente significantes. No entanto, os autores informaram que o estudo teve o tamanho da amostra relativamente pequeno; portanto, comparac, ões estatísticas formais entre os grupos ou entre as doses não foram planejadas. ${ }^{23}$

Parvizi et al., em um estudo retrospectivo de 26.415 artroplastias primárias e de revisão, feitas em sua instituição entre 2000 e 2010, recomendaram que os esforços devem ser feitos para minimizar o risco de EP durante as duas primeiras semanas após o procedimento, já que $81 \%$ dos casos documentados de EP sintomáticos ocorreram dentro de três dias de pós-operatório, $89 \%$ dentro de uma semana de pós-operatório e $94 \%$ dentro de duas semanas no pós-operatório. ${ }^{24}$

Existem alguns fatores de risco associados com a possibilidade de EP após a ATJ: quantidade total de sangramento na cirurgia, ${ }^{25}$ idade $\geq 70$, sexo feminino, maior índice de massa corporal, ${ }^{26}$ atraso na administração de tromboprofilaxia póscirúrgica $^{27}$ e em pacientes com tipagem sanguínea $A B{ }^{28}$

Em resumo, ao fazer cirurgias de ATJ o cirurgião deve estar ciente do potencial risco da ocorrência de TVP e EP. A mobilização precoce e os métodos mecânicos de prevenção podem ser usados. $\mathrm{O}$ risco e o benefício dos métodos farmacológicos devem ser discutidos com os pacientes. Se, por um lado, o objetivo é prevenir a ocorrência de TVP, inversamente, evitar complicações advindas do sangramento é essencial.

\section{Conflitos de Interesse}

Os autores declaram não haver conflitos de interesse.

\section{Referências}

1 Song K, Xu Z, Rong Z, Yang X, Yao Y, Shen Y, et al. The incidence of venous thromboembolism following total knee arthroplasty: a prospective study by using computed tomographic pulmonary angiography in combination with bilateral lower limb venography. Blood Coagul Fibrinolysis 2016;27(3):266-9

2 Blanchard J, Meuwly JY, Leyvraz PF, Miron MJ, Bounameaux H, Hoffmeyer P, et al. Prevention of deep-vein thrombosis after total knee replacement. Randomised comparison between a low-molecular-weight heparin (nadroparin) and mechanical prophylaxis with a foot-pump system. J Bone Joint Surg Br 1999;81(4):654-9

3 Lachiewicz PF, Kelley SS, Haden LR. Two mechanical devices for prophylaxis of thromboembolism after total knee arthroplasty. A prospective, randomised study. J Bone Joint Surg Br 2004;86(8): 1137-41

4 He ML, Xiao ZM, Lei M, Li TS, Wu H, Liao J. Continuous passive motion for preventing venous thromboembolism after total knee arthroplasty. Cochrane Database Syst Rev 2014;(7):CD008207

5 Barrack RL. Current guidelines for total joint VTE prophylaxis: dawn of a new day. J Bone Joint Surg Br 2012;94(11, Suppl A):3-7

6 Parvizi J, Jacovides CL, Bican O, Purtill JJ, Sharkey PF, Hozack WJ, et al. Is deep vein thrombosis a good proxy for pulmonary embolus? J Arthroplasty 2010;25(6, Suppl)138-44

7 Jacobs JJ, Mont MA, Bozic KJ, Della Valle CJ, Goodman SB, Lewis CG, et al. American Academy of Orthopaedic Surgeons clinical practice guideline on: preventing venous thromboembolic disease in patients undergoing elective hip and knee arthroplasty. J Bone Joint Surg Am 2012;94(8):746-7
8 Lotke PA, Lonner JH. The benefit of aspirin chemoprophylaxis for thromboembolism after total knee arthroplasty. Clin Orthop Relat Res 2006;452(452):175-80

9 Callaghan JJ, Warth LC, Hoballah JJ, Liu SS, Wells CW. Evaluation of deep venous thrombosis prophylaxis in low-risk patients undergoing total knee arthroplasty. J Arthroplasty 2008;23(6, Suppl 1):20-4

10 Bozic KJ, Vail TP, Pekow PS, Maselli JH, Lindenauer PK, Auerbach AD. Does aspirin have a role in venous thromboembolism prophylaxis in total knee arthroplasty patients? J Arthroplasty 2010; 25(7):1053-60

11 Parvizi J, Huang R, Restrepo C, Chen AF, Austin MS, Hozack WJ, et al. Low-Dose Aspirin Is Effective Chemoprophylaxis Against Clinically Important Venous Thromboembolism Following Total Joint Arthroplasty: A Preliminary Analysis. J Bone Joint Surg Am 2017;99(2):91-8

12 Liu F, Chu X, Huang J, Tian K, Hua J, Tong P. Administration of enoxaparin $24 \mathrm{~h}$ after total knee arthroplasty: safer for bleeding and equally effective for deep venous thrombosis prevention. Arch Orthop Trauma Surg 2014;134(5):679-83

13 Arsoy D, Giori N, Woolson S. Mechanical Compression Reduces Readmissions and Wound Complications from Low Molecular Weight Heparin after Total Hip or Knee Arthroplasty. San Diego: AAOS Annual Meeting; 2017

14 Bauer KA, Eriksson BI, Lassen MR, Turpie AG; Steering Committee of the Pentasaccharide in Major Knee Surgery Study. Fondaparinux compared with enoxaparin for the prevention of venous thromboembolism after elective major knee surgery. N Engl J Med 2001;345(18):1305-10

15 Lassen MR, Ageno W, Borris LC, Lieberman JR, Rosencher N, Bandel TJ, et al; RECORD3 Investigators. Rivaroxaban versus enoxaparin for thromboprophylaxis after total knee arthroplasty. N Engl J Med 2008;358(26):2776-86

16 Levitan B, Yuan Z, Turpie AG, Friedman RJ, Homering M, Berlin JA, et al. Benefit-risk assessment of rivaroxaban versus enoxaparin for the prevention of venous thromboembolism after total hip or knee arthroplasty. Vasc Health Risk Manag 2014;10(10):157-67

17 Bloch BV, Patel V, Best AJ. Thromboprophylaxis with dabigatran leads to an increased incidence of wound leakage and an increased length of stay after total joint replacement. Bone Joint J 2014;96-B(1):122-6

18 Yoshida RA, Yoshida WB, Rollo HA. Novos anticoagulantes orais para a profilaxia e tratamento do tromboembolismo venoso em cirurgias ortopédicas de grande porte. J Vasc Bras 2011;10(2): 145-53

19 Raskob GE, Gallus AS, Pineo GF, Chen D, Ramirez LM, Wright RT, et al. Apixaban versus enoxaparin for thromboprophylaxis after hip or knee replacement: pooled analysis of major venous thromboembolism and bleeding in 8464 patients from the ADVANCE-2 and ADVANCE-3 trials. J Bone Joint Surg Br 2012; 94(2):257-64

20 Gómez-Outes A, Terleira-Fernández AI, Suárez-Gea ML, VargasCastrillón E. Dabigatran, rivaroxaban, or apixaban versus enoxaparin for thromboprophylaxis after total hip or knee replacement: systematic review, meta-analysis, and indirect treatment comparisons. BMJ 2012;344:e3675

21 Revankar N, Patterson J, Kadambi A, Raymond V, El-Hadi W. A Canadian study of the cost-effectiveness of apixaban compared with enoxaparin for post-surgical venous thromboembolism prevention. Postgrad Med 2013;125(4):141-53

22 Fuji T, Wang CJ, Fujita S, Kawai Y, Nakamura M, Kimura T, et al. Safety and efficacy of edoxaban, an oral factor Xa inhibitor, versus enoxaparin for thromboprophylaxis after total knee arthroplasty: the STARS E-3 trial. Thromb Res 2014;134(6):1198-204

23 Turpie AG, Bauer KA, Davidson BL, Fisher WD, Gent M, Huo MH, et al; EXPERT Study Group. A randomized evaluation of betrixaban, an oral factor Xa inhibitor, for prevention of thromboembolic events after total knee replacement (EXPERT). Thromb Haemost 2009;101(1):68-76 
24 Parvizi J, Huang R, Raphael IJ, Maltenfort MG, Arnold WV, Rothman RH. Timing of Symptomatic Pulmonary Embolism with Warfarin Following Arthroplasty. J Arthroplasty 2015;30(6):1050-3

25 Miyagi J, Funabashi N, Suzuki M, Asano M, Kuriyama T, Komuro I, et al. Predictive indicators of deep venous thrombosis and pulmonary arterial thromboembolism in 54 subjects after total knee arthroplasty using multislice computed tomography in logistic regression models. Int J Cardiol 2007;119(1):90-4

26 Bohl DD, Maltenfort MG, Huang R, Parvizi J, Lieberman JR, Della Valle CJ. Development and Validation of a Risk Stratifica- tion System for Pulmonary Embolism After Elective Primary Total Joint Arthroplasty. J Arthroplasty 2016;31(9, Suppl) 187-91

27 Plante S, Belzile EL, Fréchette D, Lefebvre J. Analysis of contributing factors influencing thromboembolic events after total knee arthroplasty. Can J Surg 2017;60(1):30-6

28 Newman JM, Abola MV, Macpherson A, Klika AK, Barsoum WK, Higuera CA. ABO Blood Group Is a Predictor for the Development of Venous Thromboembolism After Total Joint Arthroplasty. J Arthroplasty 2017;32(9S):S254-8 